\title{
Microscopic models of source and sink for atomtronics
}

\author{
Andrey R. Kolovsky ${ }^{1,2}$ \\ ${ }^{1}$ Kirensky Institute of Physics, 660036 Krasnoyarsk, Russia and \\ ${ }^{2}$ Siberian Federal University, 660041 Krasnoyarsk, Russia
}

(Dated: April 28, 2017)

\begin{abstract}
We analyze the microscopic models of the particle source/sink which consists of one- or twosite Bose-Hubbard model (the system) weakly coupled to many-site Bose-Hubbard model (the reservoir). Assuming not equal filling factors for the system and reservoir, we numerically study equilibration dynamics and compare it with solution of the master equation on the reduced density matrix of the system. Necessary conditions for validity of the master equation approach are formulated.
\end{abstract}


Introduction. Recently much attention has been paid to coherent and incoherent transport of cold atoms in optical lattices - the system which carries many common features with electrons in solid crystals. Among the considered coherent phenomena are Bloch oscillations of Bose and Fermi atoms [1-5], directed transport of atoms [6, 7] and dynamical localization (band collapse) $[8,9]$ in driven lattices, Anderson's localization in disordered lattices [10, 11], and so on. The incoherent transport assumes the presence of relaxation processes. Typical examples are the collisional transport of spin-polarized Fermi atoms colliding with Bose atoms [12] and dissipative dynamics of Bose atoms in a quasi one-dimensional lattice, where one of the lattice sites is depleted by an electron beam [13]. These studies of coherent and incoherent transport help us to establish cold-atom analogs of electron devices - a new research direction known as atomtronics [14].

In narrower sense atomtronics implies cold-atom setups with source and sink, which are necessary ingredients of electronic devices. For example, the work [16] consider Bose atoms in a finite 1D lattice, where the first and last sites of the lattice are coupled to reservoirs of cold atoms. Theoretically this is done by introducing the Lindblad operators, which supply atoms into the first site and withdraw them from the last site [17]. The interior part of the system is assumed to be described by the Bose-Hubbard (BH) Hamiltonian,

$$
\widehat{H}=-\frac{J}{2}\left(\sum_{l=1}^{L-1} \hat{a}_{l+1}^{\dagger} \hat{a}_{l}+\text { h.c. }\right)+\frac{U}{2} \sum_{l=1}^{L} \hat{n}_{l}\left(\hat{n}_{l}-1\right)+\sum_{l=1}^{L} \delta_{l} \hat{n}_{l},
$$

which has proven to be an adequate model for Bose atoms in deep optical lattices. Naturally, this approach, which we shall refer to as the macroscopic model of the source and sink, leaves atom reservoirs unspecified. Furthermore, even if one formally specifies the reservoirs, it is not clear in advance to which extent the used Lindblad operators are justified. To clarify these questions we need a microscopic model of the source and sink.

In the present work we analyze one of possible microscopic models of the source/sink, which is based on BH model (1). Namely, we consider the $L$-site system, where the hopping matrix element between two selected sites is $\epsilon J$ instead of $J$. This divides the system into two subsystems and, to model the source, we assume that at $t=0$ all $N$ atoms are in the right-hand-side subsystem. We are interested in the equilibration process, i.e., in population dynamics of the left (initially empty) subsystem, which we shall call the system from now on.

One-site system. First we consider the case where the weak link is the first one. Denoting 
$\hat{a}_{2}$ by $\hat{b}$ and $\hat{a}_{1}$ by $\hat{a}$ we have

$$
\widehat{H}=\widehat{H}_{a}+\widehat{H}_{b}+\epsilon \widehat{H}_{i n t}
$$

where $\widehat{H}_{a}$ is the Hamiltonian of one-site BH model, $\widehat{H}_{b}$ the Hamiltonian of $(L-1)$-site BH model, and

$$
\widehat{H}_{i n t}=-\frac{J}{2}\left(\hat{b}^{\dagger} \hat{a}+\hat{b} \hat{a}^{\dagger}\right)
$$

In what follows we set $J=1$, i.e., all energy constants are measured in units of the hopping matrix element $J$.

For $\epsilon=0$ the matrix of Hamiltonian (2) in the Fock basis has block structure where the first block is defined by the condition that there are no atoms in the first site, the second block that there is one atom in the first site, and so on (see Fig. 1(a) below). Let us now adjust parameters of $\widehat{H}_{b}$ to ensure Quantum Chaos (QC) [20, 21]. This condition is controlled by calculating the level-spacing distribution for eigenenergies of every block, which should obey the Wigner-Dyson distribution for the Gaussian Orthogonal Ensemble of random matrices $[22,23]$. Through the paper we use $N / L \sim 1, U=0.2$, and $\left|\delta_{l}\right| \leq 0.05$, where the levelspacing distribution perfectly follows the Wigner-Dyson statistics [21]. This also implies that (almost all) eigenstates extend over the whole Hilbert space of the corresponding subspace [24], see Fig. 1(a).

Next we specify the initial conditions. As those we choose a random superposition of the eigenstates belonging to the first block in Fig. 1(a). These initial conditions ensure zero occupation of the first site and practically equal occupations $\bar{n}=N /(L-1)$ of the remaining $L-1$ sites. Finally we solve the Schrödinger equation with Hamiltonian (2) for nonzero $\epsilon$
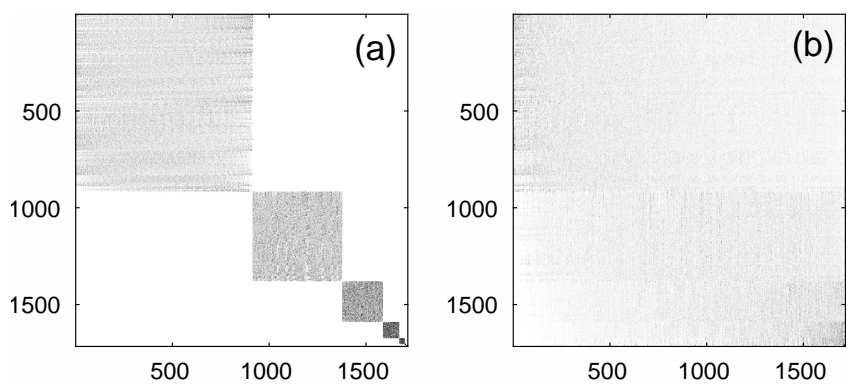

FIG. 1: Eigenstates of the total Hamiltonian for $\epsilon=0$, left panel, and $\epsilon=0.2$, right panel. The other parameters are $N=6, L=8, J=1, U=0.2$, and $\left|\delta_{l}\right| \leq 0.05$. Shown are squared modulus of expansion coefficients of the eigenstates over the Fock basis. 


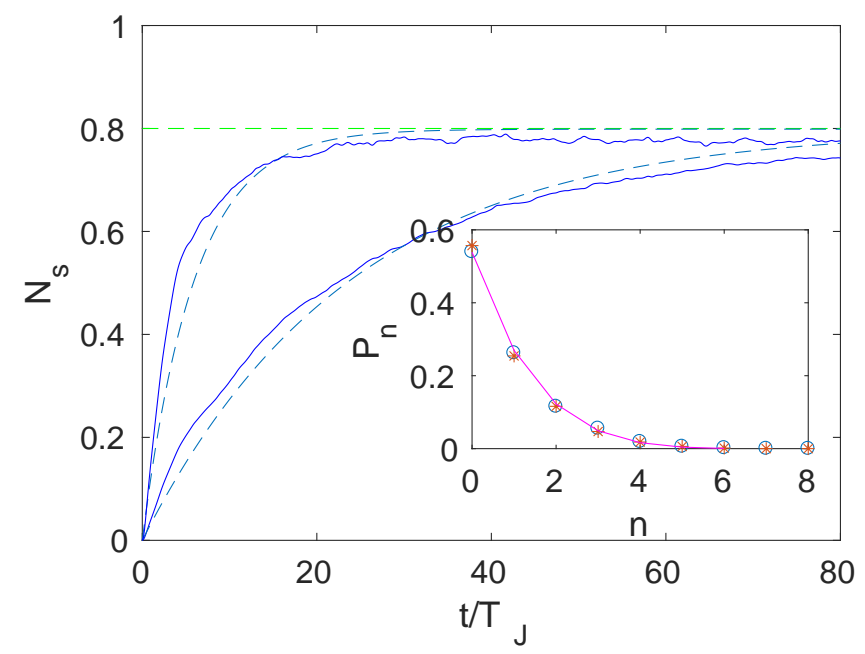

FIG. 2: The mean number of atoms in the fist site as the function of time. Parameters are $N=8$, $L=10$ (dimension of the Hilbert space $\mathcal{N}=24310$ ), and $\epsilon=0.1$, lower curve, and $\epsilon=0.2$, upper curve. The dashed lines are solutions of the master equation (6). The inset shows diagonal elements of the reduced density matrix at the end of numerical simulations, symbols, as compared to Eq. (5), solid line.

and calculate the reduced density matrix,

$$
\mathcal{R}(t)=\operatorname{Tr}_{b}\left[\mathcal{R}_{t o t}(t)\right], \quad \mathcal{R}_{t o t}(t)=|\Psi(t)\rangle\langle\Psi(t)|
$$

and the mean number of atoms $N_{s}(t)=\sum_{n=0}^{N} n \mathcal{R}_{n, n}(t)$ in the first site. We found that the reduced density matrix relaxes to a diagonal matrix, where the diagonal matrix elements $\mathcal{R}_{n, n}(t)$ approach the values

$$
P_{n}=\mathcal{N}_{n} / \mathcal{N}
$$

where $\mathcal{N}=(N+L-1) ! / N !(L-1)$ ! is the total dimension of the Hilbert space and $\mathcal{N}_{n}$ are dimensions of the corresponding subspaces (i.e., the size of blocks in Fig. 1(a). Numerical results for the mean number of atoms $N_{s}(t)$ are shown by solid lines in the main panel in Fig. 2 and validity of Eq. (5) is illustrated in the inset in Fig. 2. The observed population dynamics indicates that $\mathrm{BH}$ model can indeed serve a microscopic model of the particle source [25]. Let us also mention that Eq. (5) implies the interaction Hamiltonian (3) to couple all blocks in Fig. 1(a), so that eigenstates of the total Hamiltonian (2) extend over the whole Hilbert space, see Fig. 1(b). As mentioned above, this is reflected in the WignerDyson statistics for the energy spectrum. Thus, by analyzing the level-spacing distribution 


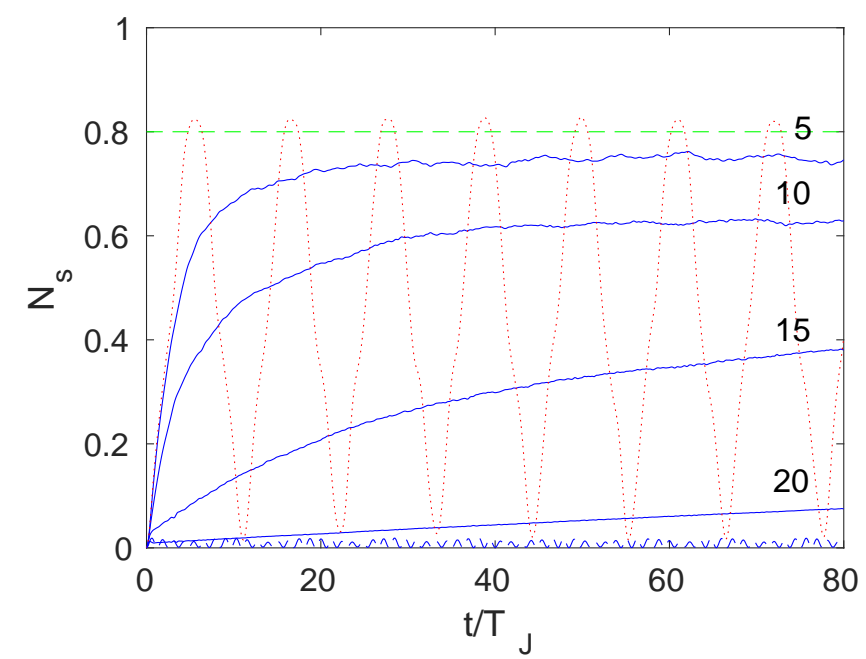

FIG. 3: The mean number of atoms in the first site for $\epsilon=0.2$ and nonzero $\delta_{1}=5,10,15,20$. Additionally, the dotted line show the case $U=0$ and the dashed line the case where the initial state of the right-hand-side subsystem is given by its ground state.

of the total Hamiltonian (which changes from Poisson to Wigner-Dyson as $\epsilon$ is increased) one finds the critical $\epsilon$ above which two subsystems do equilibrate.

To conclude this section we briefly discuss the other dynamical regimes of the system (2). First of all we mention that equilibration does not take place if we violate the conditions of QC. This is illustrated in Fig. 3, where the dotted line corresponds to the case $U=0$. Conditions of $\mathrm{QC}$ are also violated for $U \neq 0$ if we chose the initial state of the right-handside subsystem close to the energy of the ground [21]. In this case we meet completely different problem of tunneling of a Bose-Enstein condensate through an obstacle, where $N_{s}(t)$ oscillates about zero, see the dashed line in Fig. 3. For the purpose of future reference we also consider the case where the conditions of QC are satisfied but the on-site energy $\delta_{1}$ of the first site strongly deviates from zero. It is intuitively expected that this energy mismatch will suppress the equilibration process. Numerical results depicted in Fig. 3 by solid lines fully confirm this expectation.

Master equation. It is interesting to compare numerical results for the reduced density matrix $\mathcal{R}(t)$ with solutions of the master equation

$$
\begin{array}{r}
\frac{d \mathcal{R}}{d t}=-i\left[\widehat{H}_{a}, \mathcal{R}\right]+\mathcal{L}_{\text {loss }}(\mathcal{R})+\mathcal{L}_{\text {gain }}(\mathcal{R}), \\
\mathcal{L}_{\text {loss }}(\mathcal{R})=\gamma(\bar{n}+1)\left(\hat{a}^{\dagger} \hat{a} \mathcal{R}-2 \hat{a} \mathcal{R} \hat{a}^{\dagger}+\mathcal{R} \hat{a}^{\dagger} \hat{a}\right), \\
\mathcal{L}_{\text {gain }}(\mathcal{R})=\gamma \bar{n}\left(\hat{a} \hat{a}^{\dagger} \mathcal{R}-2 \hat{a}^{\dagger} \mathcal{R} \hat{a}+\mathcal{R} \hat{a} \hat{a}^{\dagger}\right),
\end{array}
$$


which is usually used to model the particle reservoirs [26-29]. In fact, providing the conditions of $\mathrm{QC}$ are satisfied, we can derive Eq. (6) from the microscopic Hamiltonian (2), in line with derivation of the master equation in Ref. [30]. Then the parameter $\bar{n}$ in the Lindblad operators has the meaning of the mean number of particles in the second site and the relaxation rate $\gamma=\epsilon^{2} \tau$, where $\tau$ is the decay time of the correlation function

$$
R\left(t-t^{\prime}\right)=\left\langle\hat{b}^{\dagger}(t) \hat{b}\left(t^{\prime}\right)\right\rangle \approx\left\langle\hat{b}^{\dagger} \hat{b}\right\rangle \exp \left(\left|t-t^{\prime}\right| / \tau\right)
$$

(here $\hat{b}^{\dagger}(t)$ and $\hat{b}\left(t^{\prime}\right)$ are the creation and anihilation operators in the interaction representation of the Hamiltonian $\widehat{H}_{b}$ ). Solutions of the master Eq. (6) are depicted in Fig. 2 by the dashed lines. Nice agreement is noticed, where small diviations are mainly due to finite size of our BH system. In particular, according to Eq. (6) the stationary values of the diadonal matrix elements obeys the relation $P_{n+1} / P_{n}=\bar{n} /(\bar{n}+1)$, while for the microscopic model we have according to Eq. (5)

$$
\frac{P_{n+1}}{P_{n}}=\frac{\bar{n}+n / L}{\bar{n}+1+(n-2) / L} .
$$

It should be stressed that Eq. (6) is not justified for the parameters of Fig. 3 because we violate one or the other assumption used to derive it. Namely, for dotted and dashed lines in Fig. 3 we violate the conditions of QC, so that the correlation function (7) does not decay. For the solid lines we violate the Markovian approximation which requires the correlation time $\tau$ to be the smallest time in the problem (in particular, smaller than $1 / \delta_{1}$ ).

Two-site system. We proceed with two-site system, where we additionally assume that the hopping matrix element between the first and second sites $(\tilde{J})$ can be varied independently of $J$. The solid lines in Fig. 4 show results of numerical simulations for different $\tilde{J}$ where, as before, we depict the mean number of atoms $N_{s}$ as the function of time. It is seen in Fig. 4 that for $\epsilon \leq \tilde{J} \leq J$ the behavior of $N_{s}$ is similar to that for the one-site system. In particular, in the stationary regime the probability $P_{n}$ to find $n$ atoms in the system is again given by the ratio $\mathcal{N}_{n} / \mathcal{N}$, see inset in Fig. 4 .

Next we address important question about degree of coherence between two sites. To answer this question we calculate the one-particle density matrix (should not be mismatched with the reduced density matrix),

$$
\rho_{l, m}^{(n)}(t)=\left\langle\Psi(t)\left|\hat{a}_{l}^{\dagger} \hat{a}_{m}\right| \Psi(t)\right\rangle
$$




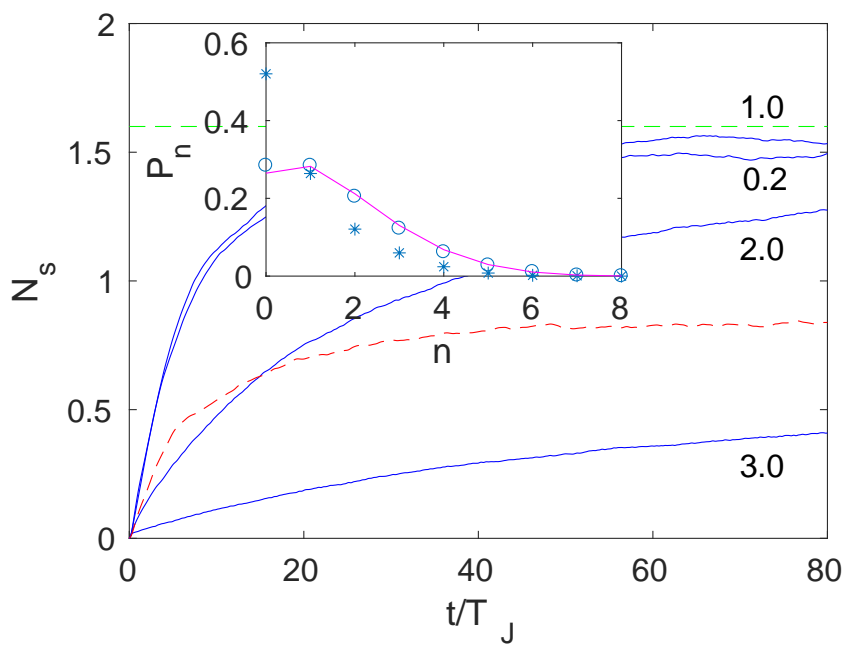

FIG. 4: The mean number of atoms in the first two sites as the function of time for different values of the hopping matrix element $\tilde{J}=0.2,1,2,3$. The other parameters are $N=8, L=10, J=1$, $U=0.2,\left|\delta_{l}\right|<0.05$, and $\epsilon=0.2$. The dashed line corresponds to the case $\tilde{J}=3$ where, however, the on-site energies for the first two sites are set to $\delta_{1}=\delta_{2}=1.5$. The open circles and asterisks in the inset show probabilities $P_{n}$ for $\tilde{J}=1$ and $\delta_{1} \approx \delta_{2} \approx 0$ and $\tilde{J}=3$ and $\delta_{1}=\delta_{2}=1.5$, respectively. The solid line is Eq. (5).

where indices $l$ and $m$ take values 1 and 2. Notice that, since the product of creation and annihilation operators conserves the number of particles, we actually have $N$ density matrices of the size $2 \times 2$, where $\operatorname{Tr}\left[\hat{\rho}^{(n)}(t)\right]$ is the probability to find $n$ particles in the system. We are mainly interested in the stationary regime when the equilibration process is over. Denoting eigenvalues of the equilibrium density matrices (9) by $\lambda_{i}^{(n)}$ and noticing that $\operatorname{Tr}\left[\hat{\rho}^{(n)}\right]=P_{n}=\lambda_{1}^{(n)}+\lambda_{2}^{(n)}$ we define the averaged weighted eigenvalues,

$$
\Lambda_{i}=\frac{1}{N} \sum_{n=1}^{N} \frac{\lambda_{i}^{(n)}}{\lambda_{1}^{(n)}+\lambda_{2}^{(n)}}, \quad \Lambda_{1}+\Lambda_{2}=1,
$$

which characterize coherence of our two-site system. It was found that for $\tilde{J} \leq J$ the matrices $\hat{\rho}^{(n)}$ are close to diagonal matrices and the quantities $\Lambda_{1} \approx \Lambda_{2} \approx 0.5$, which means that the equilibrium state of the system is incoherent.

It is commonly believed that larger $\tilde{J}$ could enhance coherence of the system. We indeed observed that for $\tilde{J}=3$ the equilibrium state is characterized by $\Lambda_{1} \approx 0.8$. However, the price we paid for the enhanced coherence is essentially longer relaxation time. (In this sense an increase of $\tilde{J}$ has the same effect as an increase of $\delta_{1}$ in the one-site problem, see solid lines 
in Fig. 3.) A way around this problem is to compensate the increase of $\tilde{J}$ by the proportional increase of the on-site energies $\delta_{1}$ and $\delta_{2}$, see dashed line in Fig. 4. Remarkably, for this setup $\Lambda_{1}$ reaches the value 0.99 , which means that the equilibrium state of the system is perfectly coherent. We also notice that probabilities $P_{n}$ now obey the exponential law, see asterisks in the inset in Fig. 4. This tell us that the currently considered setup can be mapped into a one-state bosonic system coupled to a particle reservoir, where the single-particle state of the system is given by the symmetric superposition of two Wannier functions associated with the first and second lattice sites. Needless to say that for negative $\delta_{1}=\delta_{2}=-1.5$ we have a similar situation where, however, the single-particle state is given by the antisymmetric superposition of the Wannier functions.

Conclusions. We considered microscopic model of the atom source based on the BoseHubbard Hamiltonian and compared it with the macroscopic model, which is currently used in atomtronics. In this macroscopic model atoms in a lattice obey the master equation with the Lindblad operators acting on one lattice site which is directly coupled to a reservoir of cold atoms. As the result, occupations of the lattice sites relax to some prescribed values, with no coherence between atoms in different sites (i.e., the one-particle density matrix is diagonal in the Wannier basis). We identified conditions under which the microscopic and macroscopic models give the same result, that can be viewed as justification of the macroscopic model from the first prinsiples.

The second result of the paper is demonstration of the fact that the microscopic model can show other dynamical regimes, which are not captured by the discussed macroscopic model. In particular, by adjusting the system parameters we can populate the lattice by atoms which are in a coherent superposition of the Wannier states. Furthermore, for the considered in the work example the one-particle density matrix was found to have only one macroscopic eigenvalue and, hence, the equilibrium state of the system is a perfect Bose-Einstein condensate.

We would like to emphasize that in the present work we kept atom-atom interactions as weak as possible. This leaves aside a number of self-traping effects, which are currently under active discussions in the context of atomtronics. It would be interesting to extend the presented analysis beyond the two-site system, where one can study the self-tapping effects in a systematic way.

Acknowledgements. The author acknowledges financial support from Russian Foundation 
for Basic Research, Government of Krasnoyarsk Territory, and Krasnoyarsk Region Science and Technology Support Fund through the grant No. 16-42-240746.

[1] M. Ben Dahan, E. Peik, J. Reichel, Y. Castin and C. Salomon, Bloch oscillations of atoms in an optical potential, Phys. Rev. Lett. 76, 4508 (1996).

[2] O. Morsch, J. H. Müller, M. Cristiani, D. Ciampini, and E. Arimondo, Bloch oscillations and mean-field effects of Bose-Einstein condensates in 1D optical lattices, Phys. Rev. Lett. 87, $140402(2001)$.

[3] A. R. Kolovsky and H. J. Korsch, Bloch oscillations of cold atoms in optical lattices, Int. J. of Mod. Physics B 18, 1235 (2004).

[4] M. Fattori, C. D'Errico, G. Roati, M. Zaccanti, M. Jona-Lasinio, M. Modugno, M. Inguscio, and G. Modugno, Atom interferometry with a weakly interacting Bose-Einstein condensate, Phys. Rev. Lett. 100, 080405 (2008).

[5] F. Meinert, M. J. Mark, E. Kirilov, K. Lauber, P. Weinmann, M. Gröbner, and H.-C. Nägerl, Interaction-induced quantum phase revivals and evidence for the transition to the quantum chaotic regime in $1 D$ atomic Bloch oscillations, Phys. Rev. Lett. 112, 193003 (2013).

[6] A. Alberti , V. V. Ivanov, G. M. Tino and G. Ferrari, Engineering the quantum transport of atomic wavefunctions over macroscopic distances, Nature Physics, 5, 547 (2009).

[7] E. Haller, R. Hart, M. J. Mark, J. G. Danzl, L. Reichsöllner, and H.-Ch. Nägerl, Inducing transport in a dissipation-free lattice with super Bloch oscillations, Phys. Rev. Lett. 104, $200403(2010)$.

[8] H. Lignier, C. Sias, D. Ciampini, Y. Singh, A. Zenesini, O. Morsch, and E. Arimondo, Dynamical control of matter-wave tunneling in periodic potentials, Phys. Rev. Lett. 99, 220403 (2007).

[9] A. Eckardt, M. Holthaus, H. Lignier, A. Zenesini, D. Ciampini, O. Morsch, and E. Arimondo Exploring dynamic localization with a Bose-Einstein condensate, Phys. Rev. A 79, 013611 (2009).

[10] J. Billy, V. Josse, Z. Zuo, A. Bernard, B. Hambrecht, P. Lugan, D. Clement, L. SanchezPalencia, Ph. Bouyer, and A. Aspect, Direct observation of Anderson localization of matterwaves in a controlled disorder, Nature 453, 893 (2008). 
[11] G. Roati, C. D'Errico, L. Fallani, M. Fattori, C. Fort, M. Zaccanti, G. Modugno, M. Modugno, and M. Inguscio, Anderson localization of a non-interacting Bose-Einstein condensate, Nature 453, 891 (2008).

[12] H. Ott, E. de Mirandes, F. Ferlaino, G. Roati, G. Modugno, and M. Inguscio, Collisionally induced transport in periodic potentials, Phys. Rev. Lett. 92, 160601 (2004).

[13] G. Barontini, R. Labouvie, F. Stubenrauch, A. Vogler, V. Guarrera, and H. Ott, Controlling dynamics of an open many-body quantum system with localized dissipation, Phys. Rev. Lett. 110, $035302(2013)$.

[14] Up to the best of our knowledge the term 'atomtronics' was introduced for the first time in Ref. [15].

[15] B. T. Seaman, M. Krämer, D. Z. Anderson, and M. J. Holland, Atomtronics: Ultracold-atom analogs of electronic devices, Phys. Rev. A 75, 023615 (2007).

[16] G. Kordas, D. Witthaut, and S. Wimberger, Non-equilibrium dynamics in dissipative BoseHubbard chains, Ann. Phys. (Berlin) 527, 619 (2015).

[17] We mention that similar setup is used to study transport of Fermi atoms and spin transport in spin chains, where one can solve the problem analytically Ref. [18, 19].

[18] T. Prosen, B. Żunkovich, Exact solution of Markovian master equations for quadratic Fermi systems: thermal baths, open $X Y$ spin chains and non-equilibrium phase transition, New J. of Phys. 12, 025016 (2010).

[19] T. Prosen, Exact nonequilibrium steady state of a strongly driven open XXZ chain, Phys. Rev. Lett. 107, 137201 (2011).

[20] A. R. Kolovsky and A. Buchleitner, Quantum chaos in the Bose-Hubbard model, Europhys. Lett. 68, 632 (2004).

[21] A. R. Kolovsky, Bose-Hubbard Hamiltonian: Quantum chaos approach, Int. J. of Modern Physics B 30, 1630009 (2016).

[22] M. J. Giannoni, A. Voros, and J. Zinn-Justin (Editors), Chaos and Quantum Physics, (NorthHolland, Amsterdam) 1991.

[23] H.-J. Stöckmann, Quantum Chaos, Cambridge University Press, Cambridge, 1999.

[24] This is a quantum manifestation of chaotic nature of the classical Bose-Hubbard model, where the chaotic component occupies almost whole available phase space [21].

[25] We checked that equilibration takes place also in the case where initial occupation of the first 
site exceeds $N / L$. Thus the BH model can serve a microscopic model of the sink as well.

[26] H.-P. Breuer, and F. Petruccione, The Theory of Open Quantum Systems (Oxford University Press, New York, 2007).

[27] D. Witthaut, F. Trimborn, and S. Wimberger, Dissipation induced coherence of a two-mode Bose-Einstein condensate, Phys. Rev. Lett. 101, 200402 (2008).

[28] P. Barmettler and C. Kollath, Controllable manipulation and detection of local densities and bipartite entanglement in a quantum gas by a dissipative defect, Phys. Rev. A 84, 041606(R) (2011).

[29] G. Kordas, D. Witthaut, P. Buonsante, A. Vezzani, R. Burioni, A.I. Karanikas, and S. Wimberger, The dissipative Bose-Hubbard model, Eur. Phys. J. Special Topics 224, 2127 (2015).

[30] A. R. Kolovsky, Number of degrees of freedom for a thermostat, Phys. Rev. E 50, 3569 (1994). 